Title:

A NONLINEAR MESOSCOPIC ELASTIC CLASS OF MATERIALS

Author(s):

Paul A. Johnson, ESS-4

Robert A. Guyer, EES-4 and Univ. of Mass, Amberst

Lev A. Ostrovsky, CIRES/NOAA

Submitted to:

International Symposium on Nonlinear Acoustic, Gottingen, Germany, September 1-4, 1999. Publication date March 2000

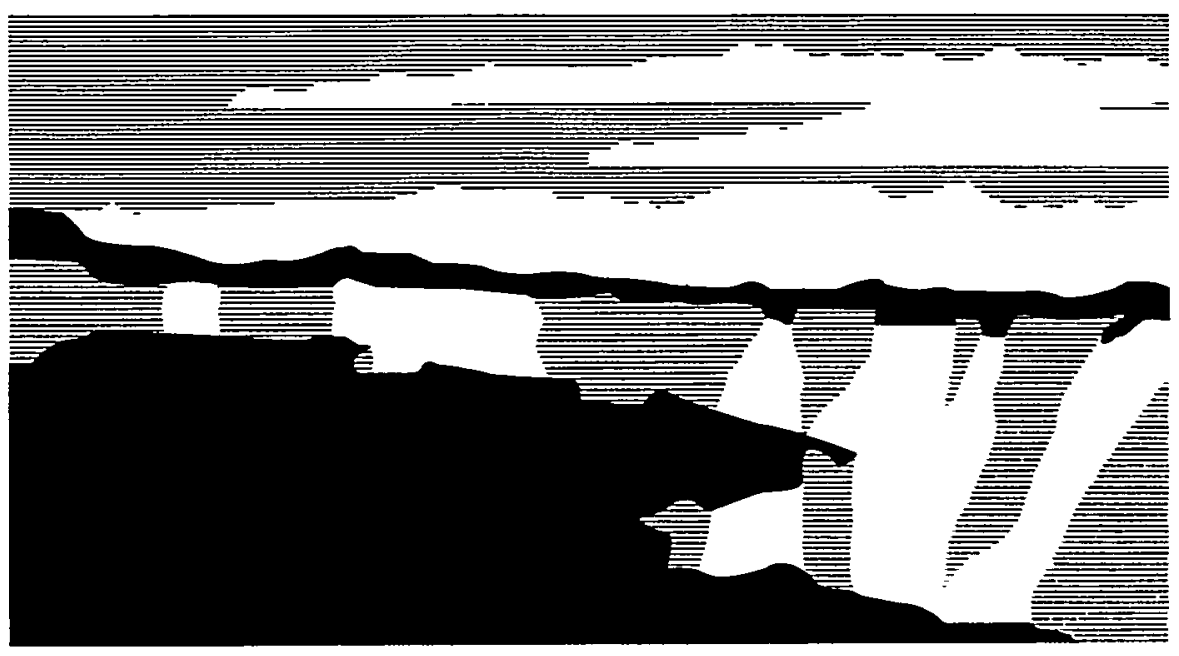

Los Alamos National Laboratory, an affirmative action/equal opportunity employer, is operated by the University of California lor the U.S. Department of Energy under contract W-7405-ENG-36. By acceptance of this article, the publisher recognizes that the U.S. Government relains a nonexclusive, royalty-free license to publish or reproduce the published form of this contribution, or to allow others to do so, for U.S. Government purposes. The Los Alamos National Laboratory requests that the publisher identify this article as work performed under the auspices of the U.S. Department of Energy. 


\section{DISCLAIMER}

This report was prepared as an account of work sponsored by an agency of the United States Government. Neither the United States Government nor any agency thereof, nor any of their employees, make any warranty, express or implied, or assumes any legal liability or responsibility for the accuracy, completeness, or usefulness of any information, apparatus, product, or process disclosed, or represents that its use would not infringe privately owned rights. Reference herein to any specific commercial product, process, or service by trade name, trademark, manufacturer, or otherwise does not necessarily constitute or imply its endorsement, recommendation, or favoring by the United States Government or any agency thereof. The views and opinions of authors expressed herein do not necessarily state or reflect those of the United States Government or any agency thereof. 


\section{DISCLAIMER}

Portions of this document may be illegible in electronic image products. Images are produced from the best available original document. 


\title{
A Nonlinear Mesoscopic Elastic Class of Materials
}

\author{
Paul A. Johnson ${ }^{1}$, Robert A. Guyer ${ }^{2}$ and Lev A. Ostrovsky ${ }^{3}$ \\ ${ }^{1}$ Mail Stop D443, Los Alamos National Laboratory, Los Alamos, New Mexico 87545, \\ http://www.ees4.lanl.gov/nonlinear/ \\ ${ }^{2}$ Dept. Physics, Univ. Massachusetts, Amherst, Massachusetts \\ ${ }^{3}$ CIRES/NOAA, Univ. Colorado Boulder Colorado 80303
}

\begin{abstract}
It is becoming clear that the elastic properties of rock are shared by numerous other materials (sand, soil, some ceramics, concrete, etc.). These materials have one or more of the following properties in common: strong nonlinearity, hysteresis in stress-strain relation, slow dynamics and discrete memory. Primarily, it is the material's compliance, the mesoscopic linkages between the rigid components, that give these materials their unusual elastic properties.
\end{abstract}

\section{INTRODUCTION}

In studying the elasticity of rock, you would find that they exhibit strong nonlinearity, they are hysteretic, they display discrete memory and they have memory effects, slow dynamics. Rocks are an example of a class of materials with unusual elastic properties, nonlinear mesoscopic elastic materials, that can include sand, soil, cement, ceramics, metals containing dislocations, and many more. All or some of the above properties are seen in the elasticity of materials that we think of as consolidated materials, i.e. materials whose primary properties are due to the process of their construction or consolidation process. For example, a sandstone is a group of grains that can be composed of quartz, for instance, bonded together with silica or calcite. It is the bond system rather than the grains themselves that control the behavior of the elastic properties. These bonds are mesoscopic in size and materials with elastic properties determined by them are called nonlinear mesoscopic elastic materials. Materials with nonlinear mesoscopic elasticity stand in contrast to aluminum, diamond, or water which have elasticity due to atomic level forces between atoms/molecules, i.e., materials with atomic elasticity. Materials with atomic elasticity are well described by the Landau theory of elasticity. Nonlinear mesoscopic elastic materials are poorly described by that theory.

Quasi-Static Nonlinearity. The most fundamental elastic properties of a material are seen in the quasi-static equation of state, the stress-strain relation- 
ship (strain is the change in length over original length $\Delta L / L$ ). In figure 1 we compare the strain $(\epsilon)$ as a function of the stress $(\sigma)$ for an atomic elastic material, Pyrex glass (Fig. 1a), and a mesoscopic elastic material, Berea sandstone (Fig 1b). We note that very similar hysteretic behavior is widely observed in other materials including metals and crystals containing dislocations, in some ceramics, and in powdered metals. A phenomenological model of the quasistatic stress-strain relationship was developed by Guyer and McCall. We call this the P-M space model.
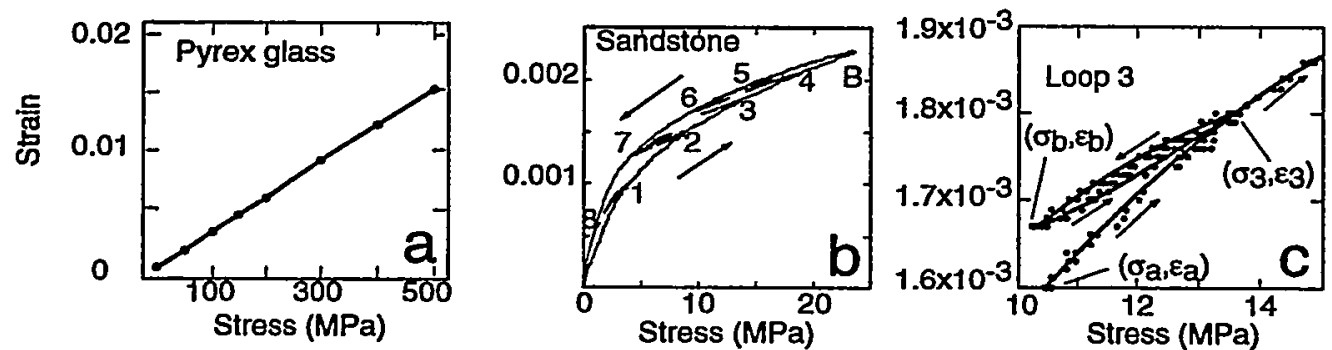

FIGURE 1. Measured stress-strain relations for Pyrex glass and Berea sandstone. From $0 \mathrm{MPa}$ to $500 \mathrm{MPa}$ the stress-strain relationship for Pyrex is linear, $d \epsilon / d \sigma$ is constant. In contrast, the sandstone is highly nonlinear, hysteretic, and contains discrete memory. When the stress of the sandstone is increased from 0 to $25 \mathrm{MPa}$ the strain increases and $d \epsilon / d \sigma$ progressively decreases. The typical rock is 2 to 3 orders of magnitude more nonlinear than the typical atomic elastic material. When the stress on the sandstone is reversed at $B$ the strain only partially reverses (most of the the strain stays in) and a hysteresis loop with cusp at $B$ develops. This loop closes when the stress is returned to 0 . The small loops at $1 \ldots 8$ in $(1 \mathrm{~b})$ come about because the stress history has 8 points at which stress makes small excursions as shown in (1b). Details of the 3rd reversal are shown in (1c). The strain traces a small hysteresis loop in the interior of the large hysteresis loop having the same sense as the big loop. When the stress reaches $\sigma_{3}$ for the second time the strain returns to the trajectory it was on before it traced the interior loop, i.e. the stress-strain trajectory of the outer loop is independent of the inner loop. This is the phenomena of discrete memory. High nonlinearity, hysteresis and discrete memory are three of the signatures of mesocopic elasticity.

Dynamic Nonlinear Elasticity. In figure 1 we see evidence for unusual elastic properties in quasi-static measurements, $\omega \rightarrow 0$. What is the dynamic elastic response of a rock? Resonant wave experiments have proved to be a valuable quantitative probe of the nonlinear elastic state of materials because of self-amplification. We term this type of experiment Nonlinear Resonant Ultrasound Spectroscopy (NRUS) after the work of those who developed linear Resonant Ultrasound Spectroscopy. In figure 2 we compare the resonance response curves of an atomic and several nonlinear mesoscopic elastic materials, and we show the strain dependencies on frequency.

The Wave Equation. In describing the observed behavior we take the following approach. The driving force for the displacement field, $u$, is developed as a power series in the strain field, $\epsilon=\partial u / \partial x$. The nonlinear terms with amplitude $\beta$, 

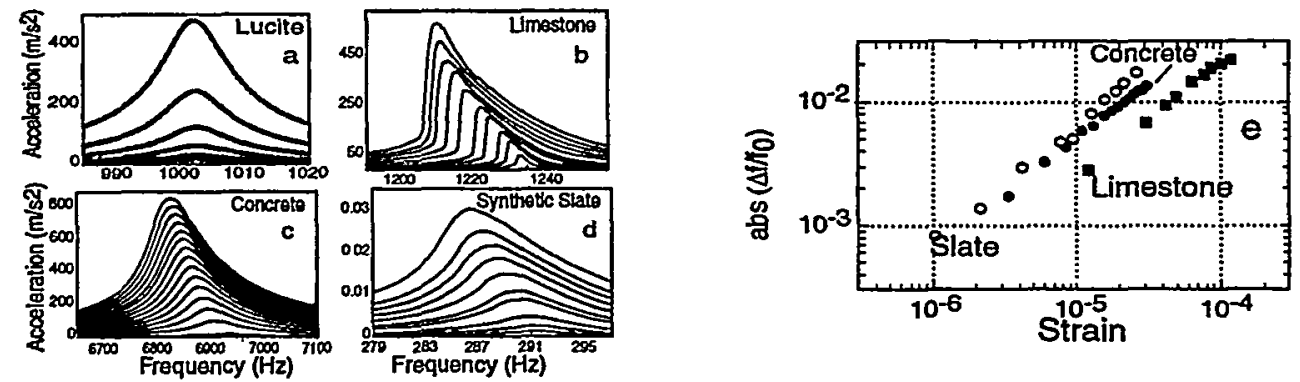

FIGURE 2. (2a) shows the response (the detected acceleration amplitude) of a bar of lucite, an atomic elastic material; (2b) shows the response of limestone; (2c) shows concrete; (2d) shows a damaged, synthetic slate [2]; and (2e) shows the nonclassical response of the change in frequency normed to the linear resonance frequency $\Delta f / f_{0}$ as a function of strain (strain is Mach number).

$\delta, \cdots$ come from traditional Landau theory. The hysteretic elastic elements in the bond system of the material make a contribution to the motion of the displacement field that depends upon $\epsilon$ and $\dot{\epsilon}$, is typically nonanalytic, and denoted by $A[\epsilon, \dot{\epsilon}]$, as seen in Figure 3.

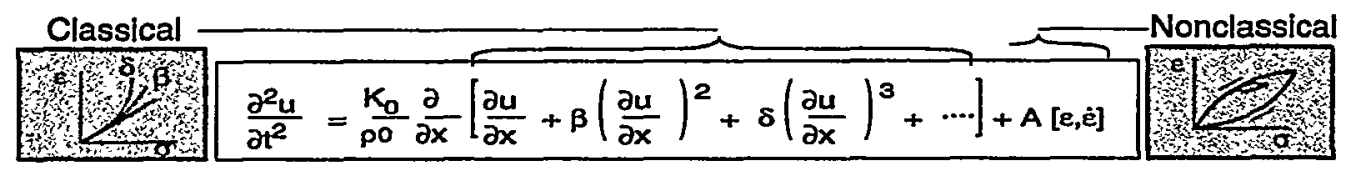

FIGURE 3. The wave equation containing classical and nonclassical (hysteretic) nonlinearity.

When the traditional theory of nonlinear elasticity is applied to a resonant bar, the nonlinear behavior is found to be due to quartic anharmonicity. For the quartic nonlinear process, 3 strain fields of amplitude $\epsilon$ and frequency $\omega$ coalesce to form a strain field of amplitude $\epsilon^{3}$ and frequency $\pm \omega \pm \omega \pm \omega=3 \omega, \omega,-\omega,-3 \omega$. Because the $\pm \omega$ components of this strain field are detected a shift in the resonant frequency proportional to $\delta \epsilon^{2}$ is expected. Instead of observing a frequency shift proportional to $\epsilon^{2}$ we see a frequency shift directly proportional to $|\epsilon|$ (e.g., Fig. 2d). We have come to realize that this unexpected result is the principal signature, in dynamics, of nonlinear mesoscopic elasticity. To understand this signature we must return to the quasi-static data shown in figure 1.

The hysteresis and discrete memory observed in quasi-static stress-strain measurements on rock are explained in terms of an assemblage of hysteretic elastic elements. When these hysteretic elastic elements are included in the description of the dynamic response of the rock, the term $A[\epsilon, \dot{\epsilon}]$ in the wave equation Figure 3, the theory of the behavior of a resonant bar yields results in qualitative and quantitative accord with experiment [1]. Thus we observe a coherent description of the elastic properties of mesoscopic materials that includes both quasistatic behavior, figure 1, and dynamic behavior, figure 2, that extends over at least 5 orders of magnitude in strain and 6 orders of magnitude in frequency. The essential feature in this coherent description is a bond system of hysteretic elastic elements. 
Slow Dynamics. As always, the story is more complicated. Careful resonant bar measurements reveal the presence of a "slow dynamics" in the elastic response of a rock , a temporary memory of large dynamic strain state. Slow dynamics is another signature of nonlinear mesoscopic elasticity. TenCate et al. [3] show that the slow dynamical response has universality characteristics as well.
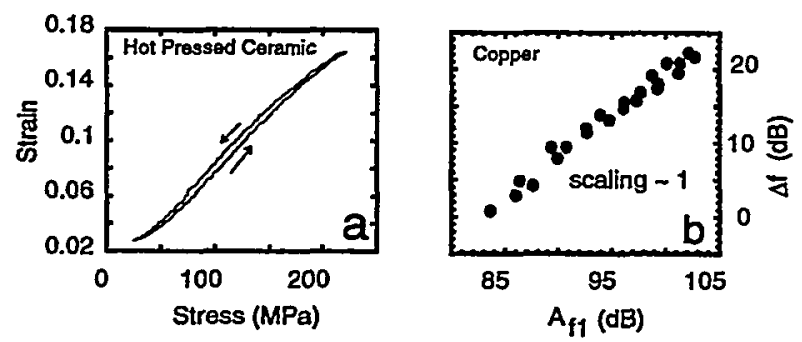

FIGURE 4. (a) Hysteretic Stress-strain relation in a ceramic containing dislocations. (b) scaling of frequency shift with strain from dynamic experiment in copper with dislocations [4].

Other Materials. The elastic properties we have illustrated with rocks characterize a class of elastic materials: rock, concrete, sand, soil, damaged materials ... All are known to share with rocks at least one of the characteristics of large nonlinearity/hysteresis/discrete memory/slow dynamics. Observations have been made in many other materials such as copper [4], in earthquake strong motion data [5] and in locally damaged materials such as ceramics, steel, aluminum and Plexiglas. Analysis of the amplitude dependence of the frequency shift and harmonic amplitude dependencies of these materials shows the signature of nonlinear mesoscopic elasticity (e.g., Figure 4) [6].

Summary. The presentation above has led us to suggest an elasticity universality class, the nonlinear mesoscopic elasticity class. There is no order parameter, symmetry, etc., to show that this supposition is correct. Rather there is the notion that the nonlinear elastic properties of these materials respond in very similar manners [7].

\section{REFERENCES}

1. Guyer, R., McCall, K., and Boitnott, G. Phys. Rev. Lett. 74, 3491 (1995).

2. Van Den Abeele, K., Carmeliet, J., and Wevers, M., this volume.

3. TenCate, J. and Shankland, T. Geophys. Res. Lett. 23, 3019 (1996); TenCate, J. Smith, D., Byers, L. and Shankland, T., this volume.

4. Nazarov, V., Ostrovsky, L., Soustova, I., and Sutin, A., Phys. Earth Planet. Int. 50, $65,(1988)$.

5. Van Den Abeele, K., Johnson, P., and Bonilla F. Bull. Seism. Soc. Am., in review (1999).

6. Johnson, P. and Sutin, A., Nonlinear Dependencies and Universality, in preparation (1999).

7. Guyer, R, and Johnson, P., Phys. Today 52, 30 (1999). 